\title{
Detection of Symptomatic Carotid Plaque Using Source Data from MR and CT Angiography: A Correlative Study
}

\author{
Ajay Gupta ${ }^{a, b}$ Hediyeh Baradaran ${ }^{a}$ Edward E. Mtui ${ }^{a}$ Hooman Kamel ${ }^{b, c}$ \\ Ankur Pandya $^{a, d}$ Ashley Giambrone ${ }^{d}$ Costantino ladecola ${ }^{b, c}$ Pina C. Sanelli ${ }^{a, d}$ \\ ${ }^{a}$ Department of Radiology, ${ }^{b}$ Feil Family Brain and Mind Research Institute, ${ }^{c}$ Department of Neurology, and ${ }^{\mathrm{d}}$ Department \\ of Healthcare Policy and Research, Weill Cornell Medical College, New York, N.Y., USA
}

\section{Key Words}

Carotid artery stenosis $\cdot$ MRA $\cdot$ CTA $\cdot$ Stroke $\cdot$ TIA

\begin{abstract}
Background: Carotid plaque MRI has been a useful method to characterize vulnerable atherosclerotic plaque elements. Recent investigations have suggested that source images from CT angiography (CTA) and MR angiography (MRA) can identify the simple high-risk features of symptom-producing carotid artery plaque. We studied the correlation and relative diagnostic accuracies of CTA and MRA source images in detecting symptomatic carotid artery plaque. Methods: Subjects were eligible if they had carotid stenosis between 50 and $99 \%$ and had MRA and CTA exams performed within 10 days of one another. We measured the soft (non-calcified) plaque and hard (calcified) plaque thickness on CTA axial source images and intraplaque high-intensity signal (IHIS) on 3D-time-of-flight MRA source images in subjects. We assessed whether a correlation existed between increasing CTA soft plaque thicknesses and the presence of MRA IHIS using the Student's t-test. We calculated the differences in sensitivity and specificity measures of CTA and MRA sourceimaging data with the occurrence of recent ipsilateral stroke or transient ischemic attack (TIA) as the reference standard. We also performed logistic regression analyses to evaluate the predictive strength of plaque showing both IHIS and in-
\end{abstract}

creased CTA soft plaque thickness in predicting symptomatic disease status. Results: Of 1994 screened patients, 48 arteries met the final inclusion criteria with MRA and CTA performed within 10 days of one another. The mean and median time between CTA and MRA exams were 2.0 days and 1 day, respectively. A total of 34 of 48 stenotic vessels (70.8\%) were responsible for giving rise to ipsilateral stroke or TIA. CTA mean soft plaque thickness was significantly greater (4.47 vs. $2.30 \mathrm{~mm}, \mathrm{p}<0.0001)$ in patients with MRA-defined IHIS, while CTA hard plaque thickness was significantly greater (2.09 vs. $1.16 \mathrm{~mm}, \mathrm{p}=0.0134$ ) in patients without MRA evidence of IHIS. CTA soft plaque thickness measurements were more sensitive than MRA IHIS (91.2 vs. $67.6 \%$, $p=0.011$ ) in detecting symptomatic plaque, while differences in specificity were not significantly different $(p=0.1573)$. In the subset of patients with both IHIS on MRA and plaque thickness $>2.4 \mathrm{~mm}$ on CTA, the odds ratio of detecting symptomatic plaque, corrected for stenosis severity, was 45.3 ( $p<$ 0.0005). Conclusions: Unprocessed source images from CTA and MRA, which are routinely evaluated for clinical studies demonstrate the highly correlated presence of IHIS and increasing soft plaque thickness. In particular, plaque that shows high-risk features on both MRA and CTA are very strongly associated with symptom-producing carotid plaque. With further validation, such techniques are promising practical methods of extracting risk information from routine neck angiographic imaging.

(c) 2015 S. Karger AG, Basel

\section{KARGER 125}

() 2015 S. Karger AG, Base

$1015-9770 / 15 / 0394-0151 \$ 39.50 / 0$
Ajay Gupta, MD

Weill Cornell Medical College

525 East 68th Street, Starr 8 A

Box 141, New York, NY 10065 (USA)

www.karger.com/ced
E-Mail ajg9004@med.cornell.edu 


\section{Introduction}

Carotid artery atherosclerosis is responsible for up to $15 \%$ of strokes in the United States and remains a significant public health burden [1]. Recent data suggest that the annual stroke risk in asymptomatic carotid artery stenosis of $\geq 50 \%$ may be on the order of $1 \%[2,3]$, making luminal stenosis alone a relatively weak risk stratification tool. Such data illustrate the need for more powerful techniques to identify vulnerable carotid artery plaque so that the asymptomatic patients at the highest risk for stroke may be targeted with more aggressive treatment, including surgical revascularization $[4,5]$. Much of the recent literature on imaging of carotid artery plaque has focused on multi-sequence carotid MRI, given its ability to accurately differentiate between high-risk elements in atherosclerotic plaque such as intraplaque hemorrhage, lipidrich necrotic core, and thinning/rupture of the fibrous cap $[6,7]$. Despite these tissue-discrimination capabilities, such MRI techniques have had only a limited impact on clinical care given the expense, time, and expertise needed to perform high-quality dedicated multi-sequence MRI.

Recent investigations have focused on characterizing features of unstable plaque from unprocessed MRA and CTA source images from which stenosis severity is already routinely determined. Intraplaque high intensity signal (IHIS) can be determined on routinely acquired axial 3D TOF MRA sources images as signal intensity $>50 \%$ compared to the background skeletal muscle, and has been studied as presumptive marker of intraplaque hemorrhage $[8,9]$. IHIS has been shown to be highly correlated with symptom-producing carotid artery plaque after controlling for stenosis severity $[8,9]$. Similarly, increasing thickness of low attenuation soft plaque as measured on CTA neck source images has been shown to be correlated with more advanced plaque as classified by multisequence carotid MRI [10] and has been shown to be highly predictive of unstable plaque responsible for recent ipsilateral ischemic symptoms [11, 12].

Given the widespread use of CTA and MRA in characterizing extracrancial carotid arterial disease, it would be useful to understand the extent to which promising simple methods of plaque imaging are correlated and can thereby identify the same high-risk tissue elements. Similarly, it would be valuable to assess how a dual-modality assessment of carotid plaque may improve the ability to predict the presence of high-risk, symptom-producing plaque. Such an understanding is particularly valuable given the paucity of correlative histopathologic data con- firming the tissue types present in regions of IHIS on MRA and soft plaque on CTA. Furthermore, such a comparison between plaque imaging modalities may provide important data needed for future studies of comparative effectiveness of simple plaque-imaging strategies in carotid disease. For these reasons, the purpose of our study was threefold: (1) to evaluate the strength of correlation between IHIS on 3D-TOF MRA source images and increasing soft plaque thickness on CTA; (2) to compare the sensitivity and specificity of these techniques in identifying symptomatic carotid artery plaque, and (3) to evaluate the predictive strength of a combined dual-modality assessment of plaque composition on CTA and MRA in detecting symptom-producing plaque.

\section{Methods}

\section{Subjects}

Our Institutional Review Board approved this protocol. Potential subject records were screened for this retrospective study via review of consecutive CTA neck examinations performed between September 1, 2009 and March 1, 2014. This database of CTA neck examinations is the same database of patients from which our group has previously identified subjects to study CTA features of carotid plaque $[11,12]$. However, the entirely unique inclusion/exclusion criteria for the current study has resulted in a cohort distinct from these prior studies with separate and new imaging analysis focused on both CTA and MRA characteristics. Subject inclusion criteria included the following: (1) moderateto high-grade extracranial ICA stenosis (50-99\%) identified on CTA neck examination as measured using NASCET criteria in the original radiologic report; (2) sufficient documentation available in the electronic medical records to confidently determine whether ipsilateral stroke or TIA had occurred in the 30 days before the CTA so that patients could be labeled as either recently symptomatic or asymptomatic (never symptomatic); (3) detailed medical record documentation of pre-existing vascular risk factors; and (4) MRA with 3D-TOF imaging performed within 10 days of the CTA examination. We applied the following exclusion criteria: (1) patients with complete extracranial carotid occlusions; (2) patients with stroke or TIA occurring greater than 30 days before the index CTA scan because these patients may have experienced relevant changes of plaque morphology between ischemic symptom onset and imaging; and (3) patients with non-diagnostic imaging in which a NASCET-style carotid artery stenosis measurement could not be provided during the initial clinical interpretation secondary to reasons such as significant motion artifact or suboptimal contrast enhancement of the arterial vasculature.

\section{CTA Imaging Technique}

The CTA neck exams in this study were performed using a standardized clinical protocol on one of several scanners at our imaging sites including Lightspeed, Pro-16, or HD-750 (GE Healthcare, Milwaukee, Wisconsin). Helical mode CT scanning was performed 
covering from the aortic arch to the $\mathrm{C} 1$ ring. Collimation was performed at $0.625 \mathrm{~mm}$, with a $\mathrm{kVp}$ of 120 and auto $\mathrm{mA}$ and a rotation time of $0.5 \mathrm{~s}$. Approximately, $90 \mathrm{ml}$ of nonionic iodinated contrast (iohexol, Omnipaque, GE Healthcare, Inc.) was administered to each patient through an 18-gauge peripheral IV at 4 to $5 \mathrm{ml} / \mathrm{s}$ using a power injector.

\section{CTA Image Analysis}

The details for CTA neck image analysis have been previously reported [10]. A board-certified neuroradiologist analyzed axial source images from the CTA exam and recorded three measurements: (1) NASCET stenosis measurements [13] using the normal distal extracranial ICA as denominator for stenosis calculation, except in cases of near-occlusion in which a stenosis value of $95 \%$ was assigned; (2) maximum thickness of the non-calcified, soft plaque component on the single axial slice on which luminal diameter stenosis was greatest; and (3) maximum thickness of the calcified, hard plaque component on the same single axial slice on which luminal diameter stenosis was greatest. These measurements were obtained on the axial slice with maximal luminal narrowing using the electronic caliper on Centricity PACS (GE Healthcare, Milwaukee, Wisconsin), which allows measurements to $0.1 \mathrm{~mm}$. Window/ level settings were set at approximately 800/200 with manual adjustments permitted to optimize discrimination between soft and hard plaque, given the inter-subject differences in contrast bolus timing and attenuation of the opacified, patent lumen. The previously reported inter-observer reliability has been shown to be high with this method with inter-reader correlation coefficients ranging from 0.91 to 0.97 for soft plaque thickness measurements [10].

\section{MRA Imaging Technique}

MRA neck studies were performed on either 1.5T or 3.0T Signa (General Electric) scanners using standard quadrature neck array coils. No dedicated high-resolution surface coils or gadolinium was administered. 3D-TOF acquisition involved a $20-\mathrm{cm}$ field-of-view centered at the carotid bifurcation, $1.4 \mathrm{~mm}$ slice thickness, and a matrix of $320 \times 192$ and $320 \times 224$ on 1.5 Tand $3 \mathrm{~T}$, respectively.

\section{MRA Imaging Analysis}

IHIS, as a presumptive marker of IPH, was determined from a method first described by Qiao et al. [9] and subsequently adapted for a subsequent study correlating plaque characteristics with symptomatic disease status [8]. Briefly, a neuroradiologist assessed the presence of hyperintense signal intensity on 3D-TOF source images using region-of-interest analysis in carotid artery plaque relative to adjacent skeletal muscle (see fig. 1,2). To ensure that both CTA and MRA studies were being analyzed on a similar axial plane, the MRA exam was evaluated for IHIS on the same craniocaudal level shown to have maximal luminal stenosis on the CTA study. To evaluate the possibility that our single slice evaluation technique may be falsely categorizing IHIS-negative studies, we reviewed the entire MRA source image data set from a randomly selected subset of 10 subjects with IHIS-negative plaque to evaluate the possibility that IHIS may have been present elsewhere over the extent of the plaque. We used a quantitative cutoff of signal intensity $50 \%$ greater than the sternocleidomastoid skeletal muscle based on the region-of-interest analysis of voxels containing IHIS. A previous report of this specific technique's reproducibility has shown inter-observer agreement rates of approximately
$80 \%$ [8]. Similarly, in studies with pathologic confirmation, diagnostic accuracy of $84 \%$ for intraplaque hemorrhage detection was found using TOF source images with excellent intra- and interobserver agreements with Kappa values ranging from 0.77 to 0.84 [9].

\section{Clinical Data Assessment}

Two study investigators blinded to imaging data determined whether each patient was either symptomatic or asymptomatic ipsilateral to the side of the index carotid artery. Symptomatic disease was defined as prior ipsilateral TIA or stroke occurring before the MRA and CTA exam. We used American Heart Association definitions of TIA and stroke [14], with a stroke and TIA defined as a permanent or transient episode, respectively, of neurological dysfunction caused by focal brain or retinal ischemia. We required that all strokes had either MR or CT imaging confirmation. We classified stroke or TIA as being symptomatic disease only when events were referable to the stenotic ICA. Days between qualifying ipsilateral ischemic events and index CTA were calculated. Asymptomatic carotid disease was defined as the absence of any prior history of stroke or TIA ipsilateral to the index carotid artery. Any disagreements in data extraction including ischemic outcome adjudication were resolved by consensus.

The same two investigators also collected preexisting vascular risk factors after a complete examination of the electronic medical record. We recorded the presence of the following risk factors: (1) a smoking history; (2) diabetes (a Hemoglobin A1C $>6.5 \%$ or on diabetic medication); (3) hypertension (blood pressure $>140 / 90$ or on antihypertensive medication); (4) atrial fibrillation; (5) hyperlipidemia (LDL $>100$ or on statin); and (6) coronary artery disease.

\section{Statistical Analysis}

Categorical variables are presented as $\mathrm{n}(\%)$ and continuous variables as mean \pm SD. Differences between patient characteristics and clinical risk factors and the presence of symptomatic versus asymptomatic plaque was evaluated with the Student's t-test or Chi-square test, where appropriate. We used the Student's t-test to determine whether the presence or absence of IHIS on MRA was associated with differences in hard and soft plaque thickness on CTA. We also performed a subset analysis stratified by magnet field strength to determine whether the presumptive markers of vulnerable plaque (IHIS and soft plaque) demonstrated a statistically significant relationship on both $1.5 \mathrm{~T}$ and $3.0 \mathrm{~T}$ platforms.

In previous investigations $[11,12]$ soft plaque thickness measurements of 3.5 and $2.2 \mathrm{~mm}$ were found to be the optimal cutoffs to predict the presence of symptomatic plaque in patients with 70-99 and 50-69\% ICA stenosis, respectively. Because of the possible correlation between increasing stenosis severity and soft plaque thickness, we used these stratified soft plaque thickness cutoffs in our analysis. We calculated operating characteristics including sensitivity, specificity, PPV, NPV, and accuracy (defined as the number of correct assessments out of all assessments) for detecting the presence of symptomatic plaque using the following definitions of a positive test result: (1) on MRA, the presence of IHIS for all stenosis severities was considered a positive test result; and (2) on CTA, soft plaque thicknesses $>3.5 \mathrm{~mm}$ in subjects with 70 to $99 \%$ stenosis and $>2.2 \mathrm{~mm}$ in 50 to $69 \%$ stenosis were considered positive test results. We assessed whether there were statistically significant differences in sensitivity and specificity be- 

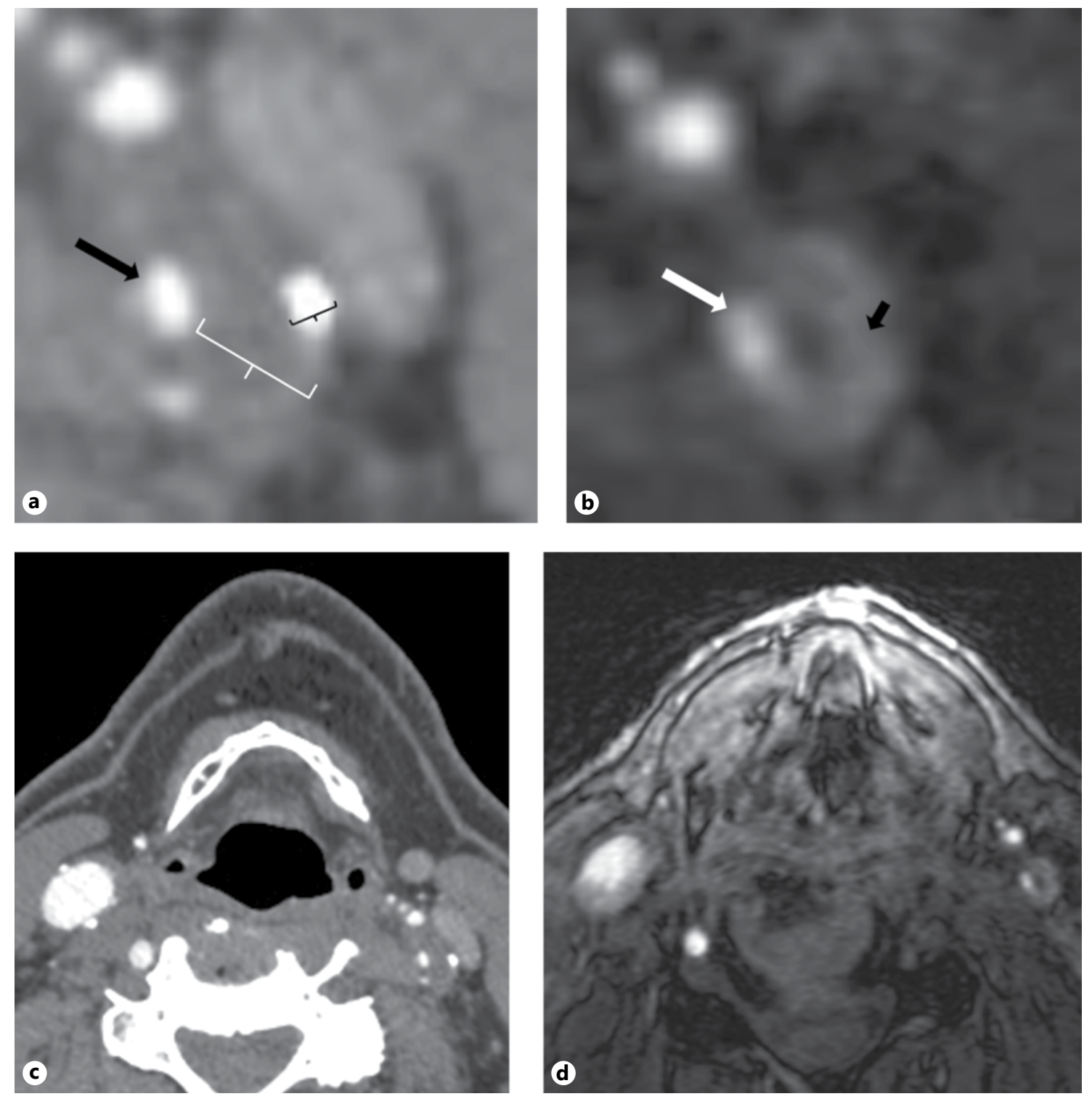

Fig. 1. a Magnified axial CTA image of the proximal left internal carotid artery in a representative subject. Black arrow represents the residual patent ICA lumen. The white calipers represent maximum linear soft plaque thickness and black calipers represent maximum hard plaque thickness measurement. b Correlative 3D-TOF source MRA image for the same subject shown in (a).

tween CTA soft plaque measurements and MRA IHIS assessments for the diagnosis of symptomatic carotid artery plaque using the $\mathrm{McNemar}$ 's test for correlated proportions. We also calculated the odds ratio expressing the association between hard plaque thickness, soft plaque thickness, and the presence of IHIS in a univariate analysis and corrected for stenosis severity in a logistic regression analysis. All $\mathrm{p}$ values are two-sided with statistical significance evaluated at the 0.05 alpha level. All analyses were performed using SAS 9.3 (SAS Institute, Cary, N.C., USA).
Patient lumen shown with the white arrow and area of IHIS is shown with the black arrow. c Larger field-of view image showing both ICAs on the same CTA source image shown in detail in (a). d Larger field-of-view image showing both ICAs on the same MRA source image shown in detail in (b).

\section{Results}

\section{Subject Characteristics}

We screened 1994 patients who had undergone CTA. We excluded 1,538 patients because these subjects did not have $\geq 50-99 \%$ extracranial internal carotid artery stenosis and 79 because of complete carotid occlusion, leaving 183 of the original 1994 still potentially eligible. Of these, 

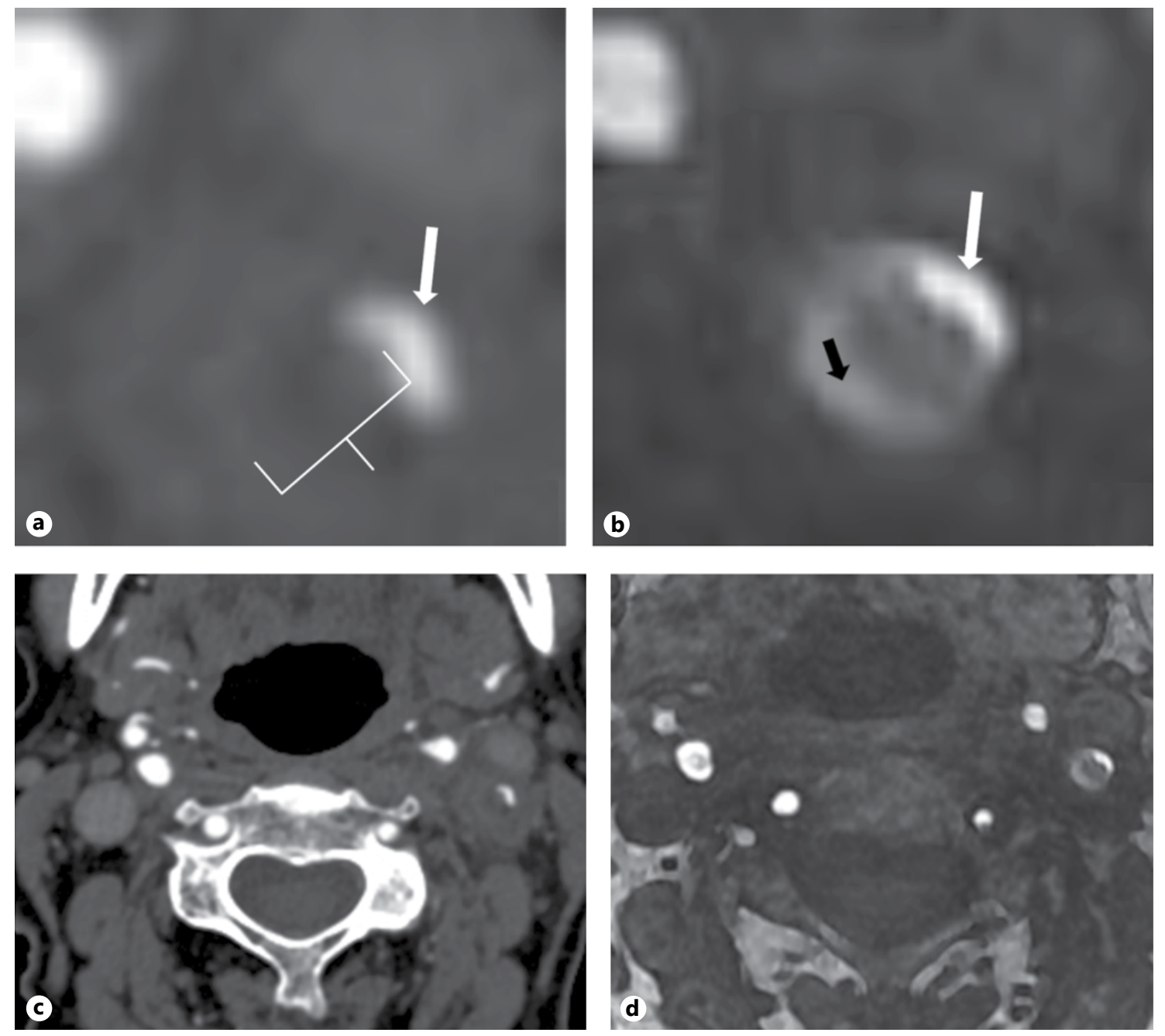

Fig. 2. a Representative magnified axial CTA image of the proximal left internal carotid artery in a subject with predominantly soft plaque. White arrow represents the residual patent ICA lumen. The white calipers represent maximum linear soft plaque thickness. This subject has no discernable hard plaque at this level. b Correlative 3D-TOF source MRA image for the same subject

48 arteries met final inclusion criteria with MRA and CTA performed within 10 days of one another. Five subjects had bilateral ICA disease eligible for analysis, yielding a total of 43 unique subjects analyzed. The mean time and median time between CTA and MRA exams were 2.0 days and 1 day, respectively. Clinical indications for CTA and MRA included suspected stroke or TIA workup in 38 subjects $(88.3 \%)$, further characterization of known carotid stenosis without suspicion of recent ischemia in 4 subjects (9.3\%), and workup of incidentally detected carotid disease detected on other brain and neck imaging in 1 subject $(2.3 \%)$.

CT Angiography and MR Angiography of Carotid Plaque shown in (a). Patient lumen shown with the white arrow and area of IHIS is shown with the black arrow. c Larger field-of view image showing both ICAs on the same CTA source image shown in detail in (a). d Larger field-of-view image showing both ICAs on the same MRA source image shown in detail in (b).

A total of 34 of 48 vessels studied (70.8\%) were from patients who had ischemic symptoms ipsilateral to the side of moderate-or high-grade carotid stenosis. Among the cerebrovascular events that had occurred, 30 had ipsilateral strokes and 4 had ipsilateral TIAs. All strokes had CT or MR imaging confirmation demonstrating infarction. All recorded ischemic events occurred within 30 days preceding the CTA index imaging study. The mean and median time between CTA imaging and the ipsilateral ischemic events were 3 days and 0 day, respectively. Patient risk factor and demographic characteristics are shown in table 1 . There was a statistically significant dif- 
ference in the degree of stenosis between symptomatic and asymptomatic subjects. The remaining risk factors were not significantly different between groups.

\section{CTA and MRA Correlative Imaging Results}

There were statistically significant differences in CTA plaque thickness measures in patients with and without MRA defined IHIS (table 2). CTA mean soft plaque thickness was significantly greater $(4.47$ vs. $2.27 \mathrm{~mm}, \mathrm{p}<$ 0.0001 ) in patients with MRA-defined IHIS, while CTA hard plaque thickness was significantly greater (2.14 vs. $1.16 \mathrm{~mm}, \mathrm{p}=0.0073$ ) in patients without MRA evidence of IHIS. The association between the presumptive highrisk markers of CTA soft plaque thickness and MRA IHIS was preserved in subset analyses across $1.5 \mathrm{~T}$ and 3.0T magnet field strengths (table 3 ). In the subset of 10 randomly selected subjects deemed to have IHIS-negative MRA on the axial slice of greatest luminal stenosis, full evaluation of the plaque extent in these patients revealed no evidence of IHIS over the entire length of the carotid artery plaque.

The presence of IHIS and increasing soft plaque thickness were both predictive of symptomatic status, while the presence of increasing hard plaque thickness was associated with asymptomatic plaque (table 4 ). We had 22 subjects with soft plaque $>2.4 \mathrm{~mm}$ and the presence of IHIS; conversely, we had 12 subjects with soft plaque $<2.4 \mathrm{~mm}$ and the absence of IHIS. The presence of both IHIS on MRA and $>2.4 \mathrm{~mm}$ soft plaque thickness conferred the greatest predictive strength for symptomatic plaque, with an OR of 45.3 (95\% CI 5.2-392.3) after correction for differences in stenosis. Similarly, the absence of IHIS and soft plaque thickness $<2.4 \mathrm{~mm}$ was very strongly associated with the lack of recent ipsilateral stroke or TIA with an OR of 0.022 (95\% CI 0.003-0.191) after correction for differences in stenosis.

\section{Diagnostic Accuracy of MRA and CTA in Detecting Symptomatic Plaque}

We found that dichotomized CTA soft plaque thickness (2.2 $\mathrm{mm}$ for $50-69 \%$ and $3.5 \mathrm{~mm}$ for $70-99 \%)$ was a more accurate marker of recently symptomatic plaque than MRA IHIS in both the moderate (accuracy of 82.6 vs. $69.6 \%$ ) and high-grade stenosis categories (accuracy 84.0 vs. $68.0 \%$ ) (table 4 ). When using a single optimal threshold value of $2.4 \mathrm{~mm}$ of soft plaque derived from the cohort in this study, soft plaque measurements also demonstrated greater diagnostic accuracy (91.2 vs. 67.6\%) (table 5). Moreover, McNemar's test for correlated proportions demonstrated that CTA soft plaque thickness
Table 1. Cohort demographics and risk factor differences between main study groups

\begin{tabular}{lcccc}
\hline \multirow{2}{*}{$\begin{array}{l}\text { Patient } \\
\text { characteristics and } \\
\text { risk factors }\end{array}$} & $\begin{array}{l}\text { Overall } \\
(\mathrm{n}=48)\end{array}$ & Symptoms & $\mathrm{p}$ \\
\cline { 3 - 4 } & & yes $(\mathrm{n}=34)$ & no $(\mathrm{n}=14)$ & \\
\hline Age, mean (years) & $74.5 \pm 9.8$ & $74.5 \pm 10.2$ & $74.4 \pm 9.0$ & 0.9639 \\
Sex, male & $28(58.3)$ & $21(61.8)$ & $7(50.0)$ & 0.4524 \\
Stenosis, \% & $72.9 \pm 15.0$ & $76.7 \pm 14.9$ & $63.8 \pm 11.0$ & 0.0055 \\
Hypertension & $41(85.4)$ & $29(85.3)$ & $12(85.7)$ & 1.0000 \\
Diabetes & $13(27.1)$ & $10(29.4)$ & $3(21.4)$ & 0.7279 \\
Hyperlipidemia & $35(72.9)$ & $25(73.5)$ & $10(71.4)$ & 1.0000 \\
A-fib & $10(20.8)$ & $6(17.7)$ & $4(28.6)$ & 0.4480 \\
CAD & $5(10.4)$ & $4(11.8)$ & $1(7.1)$ & 1.0000 \\
Smoking history & $29(60.4)$ & $21(61.8)$ & $8(57.1)$ & 1.0000 \\
Heart failure & $3(6.3)$ & $1(2.9)$ & $2(14.3)$ & 0.1999 \\
COPD & $3(6.3)$ & $2(5.9)$ & $1(7.1)$ & 1.0000 \\
CKD & $1(2.1)$ & $0(0)$ & $1(7.1)$ & 0.2917 \\
\hline
\end{tabular}

Mean values are shown with the number of subjects shown in parenthesis and standard deviations shown after \pm signs.

Table 2. Correlation between the presence of MRA IHIS and CTA plaque thickness measurements

\begin{tabular}{lrrr}
\hline & \multicolumn{2}{l}{ MRA IHIS present? } & $\mathrm{p}$ \\
\cline { 2 - 3 } & yes $(\mathrm{n}=27)$ & no $(\mathrm{n}=21)$ & value \\
\hline CTA soft plaque thickness, mm & $4.47 \pm 1.41$ & $2.3 \pm 1.58$ & $<0.0001$ \\
CTA hard plaque thickness, mm & $1.16 \pm 1.14$ & $2.09 \pm 1.36$ & 0.0134 \\
\hline
\end{tabular}

Table 3. Correlation between the presence of MRA IHIS stratified by magnet field strength and CTA soft plaque thickness measurements

\begin{tabular}{clll}
\hline & \multicolumn{2}{l}{ MRA IHIS present? } & p value \\
\cline { 2 - 3 } & yes & no & \\
\hline $\begin{array}{c}\text { Studied on 1.5T MRI } \\
\text { CTA soft plaque } \\
\text { thickness, mm }\end{array}$ & $\begin{array}{l}4.53 \pm 1.66 \\
(\mathrm{n}=15)\end{array}$ & $\begin{array}{l}2.84 \pm 1.84 \\
(\mathrm{n}=11)\end{array}$ & 0.0261 \\
$\begin{array}{c}\text { Studied on 3T MRI } \\
\text { CTA soft plaque } \\
\text { thickness, mm }\end{array}$ & $4.39 \pm 1.07$ & $1.83 \pm 1.20$ & $<0.0001$ \\
\hline
\end{tabular}

measurements were more sensitive than MRA IHIS (91.2 vs. $67.6 \%, \mathrm{p}=0.011)$ in detecting symptomatic plaque, while differences in specificity (85.7\% for CTA soft plaque vs. $71.4 \%$ for MRA IHIS) were not statistically significant $(\mathrm{p}=0.1573)$. 
Table 4. Univariate and Multivariate logistic regression analyses showing the association (odds ratio) between each imaging marker and the risk of ipsilateral symptomatic disease

\begin{tabular}{|c|c|c|c|c|}
\hline & \multicolumn{2}{|l|}{ Univariate } & \multicolumn{2}{|l|}{ Multivariate* } \\
\hline CTA soft plaque thickness (per $1 \mathrm{~mm}$ increase) & $2.76(1.52-5.04)$ & 0.0009 & $2.42(1.30-4.53)$ & 0.0055 \\
\hline MRA presence of IHIS & $5.23(1.34-20.45)$ & 0.0175 & $6.67(1.42-31.26)$ & 0.0160 \\
\hline Soft plaque $>2.4 \mathrm{~mm}$ and presence of IHIS & $55.0(6.8-448.1)$ & 0.0002 & $45.3(5.2-392.3)$ & 0.0005 \\
\hline
\end{tabular}

* Adjusted for stenosis (\%).

Table 5. Diagnostic operating characteristics for CTA plaque thickness measurements and MRA IHIS

\begin{tabular}{|c|c|c|c|c|c|c|}
\hline Overall & 48 & & & & & \\
\hline Soft (2.4 mm threshold) & & $91.2(76.3-98.1)$ & $85.7(57.2-98.2)$ & $93.9(79.8-99.3)$ & $80.0(51.9-95.7)$ & $89.6(77.3-96.5)$ \\
\hline IHIS & & $67.6(49.5-82.6)$ & $71.4(41.9-91.6)$ & $85.2(66.3-95.8)$ & $47.6(25.7-70.2)$ & $68.8(53.7-81.3)$ \\
\hline $50-69 \%$ stenosis & 23 & & & & & \\
\hline 70-99\% stenosis & 25 & & & & & \\
\hline Soft (3.5 mm threshold) & & $81.8(59.7-94.8)$ & $100.0(29.2-100.0)$ & $100.0(81.5-100.0)$ & $42.9(9.9-81.6)$ & $84.0(63.9-95.5)$ \\
\hline IHIS & & $63.6(40.7-82.8)$ & $100.0(29.2-100.0)$ & $100.0(76.8-100.0)$ & $27.3(6.0-61.0)$ & $68.0(46.5-85.1)$ \\
\hline Soft $(2.4 \mathrm{~mm})$ and IHIS & 36 & $91.7(73.0-99.0)$ & $83.3(51.6-97.9)$ & $91.7(73.0-99.0)$ & $83.3(51.6-97.9)$ & $88.9(73.9-96.9)$ \\
\hline
\end{tabular}

All values shown in the table expressed as percentages.

\section{Discussion}

By using only the unprocessed source data from CTA and MRA imaging of carotid artery stenosis, we found that increasing CTA soft plaque thickness measurements were strongly associated with the presence of IHIS on MRA source images. Moreover, using the presence of symptomatic plaque as a reference standard, we found a statistically and significantly higher sensitivity of CTA soft plaque measurements compared to MRA IHIS. Perhaps of greatest interest, we found that a dual-modality assessment of carotid plaque may identity those plaques most likely to be symptom-producing, as plaques with both IHIS on MRA and soft plaque thickness of greater than $2.4 \mathrm{~mm}$ were very strongly associated with recent ipsilateral stroke or TIA. Unlike other studies evaluating the correlation between high-risk plaque features on MRI and CTA $[9,10]$, ours was the first study to use MRI techniques without gadolinium, a carotid coil, or any imaging sequences beyond those needed for a 3D-TOF acquisition. Similarly, CTA exams were done using a routine standard of care clinical protocol and all image analysis was performed without additional post-processing or specialized software.

The high correlation between IHIS and plaque soft thickness measurements on CTA may be related to the imaging appearance of lipid or hemorrhage known to be present in vulnerable atherosclerotic plaque. Recent pooled data from prospective observational studies of carotid stenosis patients after carotid plaque MRI demonstrates that intraplaque hemorrhage and lipid-rich necrotic core are two important tissue types that can confer a 3- to 6-fold increased risk of ischemic events for a given degree of stenosis [6]. It is likely that the areas of low plaque attenuation on CT and areas of bright signal on MR represent varying degrees of lipid-rich necrotic core and intraplaque hemorrhage and may therefore represent simple composite imaging markers of 
these relatively high-risk atherosclerotic plaque elements. The precise differentiation between lipid and hemorrhage in these plaques is likely not possible on CTA or MRA source images alone. Hounsfield unit attenuation measurements have historically shown a poor ability to discriminate [15-18] between specific tissue types in advanced atherosclerotic plaque. Similarly, without a dedicated multisequence protocol or a carotid coil, there will likely be similar limitations on the accuracy of 3D-TOF MRA images in differentiating tissue elements which cause T1-shortening, such as lipid or methemoglobin within intraplaque hemorrhage [8]. However, given the relatively similar stroke risk conferred by the presence of plaque hemorrhage and lipidcore, the clinical value of differentiating these tissue types may be limited. Ultrasound data also support this approach of using a simplified marker of risk rather than using imaging to differentiate between tissue histologies, as several investigations have shown echolucent plaque [19-21] to be a high-risk marker of carotid stenosis more likely to cause symptoms than predominantly echogenic plaque.

The presence of increasing hard plaque thicknesses on CTA was associated with the absence of IHIS on MRA. The physical basis of this finding likely is related to the marked hypointensity generally seen with dense calcification on MRI images. As prior reports including a systematic review of the role of calcium deposition in carotid plaque stability have suggested $[22,23]$, increasing volumes of calcium in carotid atherosclerosis likely indicate relative plaque stability for a given degree of stenosis. The mechanism by which calcium may improve plaque stability requires further elucidation, but may in part be related to the greater mechanical stability afforded by calcium deposition or relatively antithrombotic property of calcified plaque compared to soft tissue plaque [24]. Alternatively, it could be the case that plaque calcification plays little role in plaque stability but may instead represent an epiphenomenon seen only in plaque stable long enough to allow for the physiologic deposition of calcium. Since the detection of calcium is most easily made on CT, the absence of IHIS or MRA is likely not an accurate indicator of relatively stable carotid plaque as has been suggested with increasing hard plaque thickness on CTA.

In our study, we also demonstrated that CTA soft plaque thickness demonstrated significantly higher sensitivity for the detection of symptomatic plaque. Though the ultimate validation of these methods will be the prediction of future ischemic events, our data is still infor- mative, as it gives us insight into the potential relative comparative accuracy of simple plaque imaging techniques. MRA-based IHIS determination is dichotomous, while CTA plaque thickness measurements are potentially more informative as continuous variables, which can allow for specific positivity thresholds depending on the severity of stenosis. Moreover, the significantly higher spatial resolution of CTA source images compared with 3D-TOF MRA imaging obtained without a dedicated carotid coil may also explain the relatively higher reproducibility measures seen in previous reports of these CTA techniques [10]. Speed and accessibility in the acute setting are additional benefits of the CTA imaging strategy. Nonetheless, the optimal simple plaque imaging strategy (including possibly a dual or multi-modality approach) will require further study, as MRA has several factors in its favor including potentially more valuable brain imaging information from concurrently acquired brain MRI, the lack of ionizing radiation, and no need for intravenous contrast administration.

We believe that our current study adds two main important advances in our understanding carotid artery disease. First, this study shows that high-risk features of carotid plaque are evident using standard cross-sectional imaging techniques on both MRI and CT without advanced imaging equipment. Though there are several papers showing the value of source MRA $[9,25,26]$ and CTA imaging $[17,18,27-30]$ in carotid plaque, the correlation between these commonly acquired imaging tests has not been studied formally. The high correlation between IHIS on MRA and soft plaque on CTA suggests that there is significant overlap in the appearance of vulnerable atherosclerotic elements across modalities. Though multi-sequence MRI of carotid plaque with a dedicated carotid coil has provided a tremendous improvement in our understanding human atherosclerosis, we believe that the challenges of performing this technique in clinical practice make the simple markers of plaque vulnerability described in this study attractive imaging biomarkers for stroke risk prediction. Second, given the decreasing risk of annual stroke in asymptomatic carotid stenosis $[3,31]$, single risk markers may not be sufficient to identify those patients at highest risk of stroke and therefore may be inadequate as a tool to inform decisions to pursue surgical revascularization [32, 33]. Toward this end, our study has identified a new potential dual-modality marker of high-risk plaque (the composite presence of IHIS on MRA and soft plaque thickness of greater than $2.4 \mathrm{~mm}$ ), which now warrants 
investigation in prospective studies. With further validation, these simple source image strategies for stroke risk stratification may allow us to obtain detailed plaque characteristics and quantitative brain hemodynamics in the same imaging platform, thereby creating an opportunity for an integrated pathophysiologic approach [34] to stroke risk stratification in carotid stenosis. To-date, such an integrated hemodynamic and plaque vulnerability risk assessment approach has been difficult to implement alongside lengthy multi-sequence carotid plaque imaging techniques.

Our study has several limitations. First, in this study, we retrospectively correlated plaque characteristics with recent ischemic events that occurred prior to $\mathrm{CT}$ or MR imaging. Prospective validation in a longitudinal cohort is needed to validate these techniques as risk-stratification techniques. However, given the converging evidence [6] confirming the prediction power of intraplaque hemorrhage and lipid-rich necrotic core, we hypothesize that the presumptive risk-markers studied would likely function similarly in predicting future events. Second, since atherosclerosis is a dynamic process, it is possible that the plaque elements we studied evolved between the time of the CTA and MRA exam. We minimized this risk by limiting our study only to those cases in which CTA and MRA were done within 10 days of one another with the mean time between imaging studies closer to 2 days in our study. Moreover, longitudinal MRI data of carotid plaque suggests that high-risk elements such as plaque hemorrhage do not change significantly over a 1-year follow-up period and can predict stroke risk for at least 5 years in prospective observational studies $[35,36]$. Third, we focused on detecting IHIS on a single slice shown to have maximal luminal stenosis on CTA, and we thereby may have not detected IHIS proximal or distal to the site of maximum stenosis. Such an approach, we believe, however, was necessary to closely co-register images and best allow us to evaluate the same tissue across modalities and explore the hypothesis that CT and MRI source-imaging data may contain similar plaque vulnerability information. Furthermore, in our subset of randomly selected IHIS-negative patients, we found that a full evaluation of the plaque extent in these patients revealed no evidence of IHIS over the entire length of the carotid artery plaque, suggesting that analyzing images at the level of maximal stenosis may be a reasonable approach to identify the presence of high-risk plaque elements within a given carotid plaque. Fourth, our study lacked histopathologic confirmation of the substrate composing IHIS on MRA and soft plaque on CTA. Despite this, there is strong evi- dence from existing histopathologic studies [26] suggesting that our presumptive imaging biomarkers likely represent varying degrees of intraplaque hemorrhage and lipid-rich necrotic core. We do agree that correlation with carotid endarterectomy specimens would be informative to enhance our understanding of the pathophysiologic features captured by these imaging strategies. Fifth, given the predominance of CT and MR performed in our local practice for the work up of suspected TIA or stroke, we lacked ultrasound plaque data on these patients which will be important to include in future multi-modality investigations of high-risk plaque features. In addition to characterizing simple high-risk features of plaque composition such as echolucency, ultrasound is also valuable, as it can provide insight into local flow hemodynamics, which is typically not available from the structural data present on MRA and CTA.

\section{Conclusions}

In conclusion, in this correlative study we were able to demonstrate a strong association between markers of presumed high-risk plaque on both CTA and MRA, and showed that CTA soft plaque measurements were more sensitive than MRA IHIS in diagnosing the presence of symptomatic plaque. Most importantly, plaque that showed high-risk features on both CTA and MRA were the most strongly predictive of symptomatic plaque. Further assessment of these simple CT and MRA measures of high-risk plaque, including in a combined approach, are now warranted in appropriately powered prospective outcome studies.

\section{Ethical Standards and Patient Consent}

We declare that all human and animal studies have been approved by the Institutional Review Board and have therefore been performed in accordance with the ethical standards laid down in the 1964 Declaration of Helsinki and its later amendments. As this was a retrospective study of existing clinical data, our IRB waived the requirement for informed consent.

\section{Author Contributions}

A.G. - Project development, funding, data analysis, manuscript writing; H.B., E.E.M., A.P. - Data collection and analysis; H.K. - Clinical data collection, critical review and writing of manuscript; C.L., P.C.S. - Study design, critical review and writing of manuscript; A.E.G. - Statistical analyses and data analysis. 


\section{Grant Support}

Dr. Gupta's effort for this project has been in part supported by the Association of University Radiologists General Electric Radiology Research Academic Fellowship (GERRAF) and the 2014-2015 Foundation of the American Society of Neuroradiology Scholar Award.

\section{Disclosure Statement}

A.G.'s effort for this project has been in part supported by the Association of University Radiologists General Electric Radiology Research Academic Fellowship (GERRAF) and the Foundation of the American Society of Neuroradiology Scholar Award. The remaining authors have no conflicts of interest relevant to this article.

\section{References}

1 Grotta JC: Clinical practice. Carotid stenosis. N Engl J Med 2013;369:1143-1150.

-2 Raman G, Moorthy D, Hadar N, Dahabreh IJ, O'Donnell TF, Thaler DE, Feldmann E, Lau J, Kitsios GD: Management strategies for asymptomatic carotid stenosis: a systematic review and meta-analysis. Ann Intern Med 2013;158:676-685.

-3 Hadar N, Raman G, Moorthy D, O'Donnell TF, Thaler DE, Feldmann E, Lau J, Kitsios GD, Dahabreh IJ: Asymptomatic carotid artery stenosis treated with medical therapy alone: temporal trends and implications for risk assessment and the design of future studies. Cerebrovasc Dis 2014;38:163-173.

$\checkmark 4$ Chalela JA: Evaluating the carotid plaque: going beyond stenosis. Cerebrovasc Dis 2009; 27(suppl 1):19-24.

$\checkmark 5$ Lanza G, Setacci C, Cremonesi A, Ricci S, Inzitari D, de Donato G, Castelli P, Pratesi C, Peinetti F, Lanza J, Zaninelli A, Gensini GF: Carotid artery stenting: second consensus document of the ICCS/ISO-SPREAD joint committee. Cerebrovasc Dis 2014;38:77-93.

6 Gupta A, Baradaran H, Schweitzer AD, Kamel H, Pandya A, Delgado D, Dunning A, Mushlin AI, Sanelli PC: Carotid plaque MRI and stroke risk: a systematic review and metaanalysis. Stroke 2013;44:3071-3077.

-7 Mono ML, Karameshev A, Slotboom J, Remonda L, Galimanis A, Jung S, Findling O, De Marchis GM, Luedi R, Kiefer C, Stuker C, Mattle HP, Schroth G, Arnold M, Nedeltchev $\mathrm{K}$, El-Koussy M: Plaque characteristics of asymptomatic carotid stenosis and risk of stroke. Cerebrovasc Dis 2012;34:343-350.

8 Gupta A, Baradaran H, Kamel H, Mangla A, Pandya A, Fodera V, Dunning A, Sanelli PC: Intraplaque high-intensity signal on $3 \mathrm{D}$ timeof-flight MR angiography is strongly associated with symptomatic carotid artery stenosis. AJNR Am J Neuroradiol 2013;35:557-561.

-9 Qiao Y, Etesami M, Malhotra S, Astor BC, Virmani R, Kolodgie FD, Trout HH 3rd, Wasserman BA: Identification of intraplaque hemorrhage on MR angiography images: a comparison of contrast-enhanced mask and time-of-flight techniques. AJNR Am J Neuroradiol 2011;32:454-459.

-10 Trelles M, Eberhardt KM, Buchholz M, Schindler A, Bayer-Karpinska A, Dichgans M, Reiser MF, Nikolaou K, Saam T: CTA for screening of complicated atherosclerotic ca- rotid plaque - American Heart Association type VI lesions as defined by MRI. AJNR Am J Neuroradiol 2013;34:2331-2337.

11 Gupta A, Baradaran H, Kamel H, Pandya A, Mangla A, Dunning A, Marshall RS, Sanelli PC: Evaluation of computed tomography angiography plaque thickness measurements in high-grade carotid artery stenosis. Stroke 2014;45:740-745.

12 Gupta A, Mtui EE, Baradaran H, Salama G, Pandya A, Kamel H, Giambrone A, Sanelli PC: CT angiographic features of symptomproducing plaque in moderate-grade carotid artery stenosis. AJNR Am J Neuroradiol 2014; 35:1714-1720.

13 Fox AJ: How to measure carotid stenosis. Radiology 1993;186:316-318.

14 Easton JD, Saver JL, Albers GW, Alberts MJ, Chaturvedi S, Feldmann E, Hatsukami TS, Higashida RT, Johnston SC, Kidwell CS, Lutsep HL, Miller E, Sacco RL; American Heart Association; American Stroke Association Stroke Council; Council on Cardiovascular Surgery and Anesthesia; Council on Cardiovascular Radiology and Intervention; Council on Cardiovascular Nursing; Interdisciplinary Council on Peripheral Vascular Disease: Definition and evaluation of transient ischemic attack: a scientific statement for healthcare professionals from the American Heart Association/ American Stroke Association Stroke Council; Council on Cardiovascular Surgery and Anesthesia; Council on Cardiovascular Radiology and Intervention; Council on Cardiovascular Nursing; and the Interdisciplinary Council on Peripheral Vascular Disease. The American Academy of Neurology affirms the value of this statement as an educational tool for neurologists. Stroke 2009;40:2276-2293.

15 de Weert TT, Cretier S, Groen HC, Homburg P, Cakir H, Wentzel JJ, Dippel DW, van der Lugt A: Atherosclerotic plaque surface morphology in the carotid bifurcation assessed with multidetector computed tomography angiography. Stroke 2009;40:1334-1340.

16 Walker LJ, Ismail A, McMeekin W, Lambert $\mathrm{D}$, Mendelow AD, Birchall D: Computed tomography angiography for the evaluation of carotid atherosclerotic plaque: correlation with histopathology of endarterectomy specimens. Stroke 2002;33:977-981.

17 U-King-Im JM, Fox AJ, Aviv RI, Howard P, Yeung R, Moody AR, Symons SP: Character- ization of carotid plaque hemorrhage: a CT angiography and $\mathrm{MR}$ intraplaque hemorrhage study. Stroke 2010;41:1623-1629.

18 Wintermark M, Jawadi SS, Rapp JH, Tihan T, Tong E, Glidden DV, Abedin S, Schaeffer S, Acevedo-Bolton G, Boudignon B, Orwoll B, Pan X, Saloner D: High-resolution CT imaging of carotid artery atherosclerotic plaques. AJNR Am J Neuroradiol 2008;29:875-882.

19 Silvestrini M, Altamura C, Cerqua R, Pasqualetti P, Viticchi G, Provinciali L, Paulon L, Vernieri F: Ultrasonographic markers of vascular risk in patients with asymptomatic carotid stenosis. J Cereb Blood Flow Metab 2013;33:619-624

20 Topakian R, King A, Kwon SU, Schaafsma A, Shipley M, Markus HS; ACES Investigators: Ultrasonic plaque echolucency and emboli signals predict stroke in asymptomatic carotid stenosis. Neurology 2011;77:751-758.

21 Hashimoto H, Tagaya M, Niki H, Etani H: Computer-assisted analysis of heterogeneity on B-mode imaging predicts instability of asymptomatic carotid plaque. Cerebrovasc Dis 2009;28:357-364.

22 Hashimoto H, Tagaya M, Niki H, Etani H: Computer-assisted analysis of heterogeneity on B-mode imaging predicts instability of asymptomatic carotid plaque. Cerebrovasc Dis 2009;28:357-364.

23 Kwee RM: Systematic review on the association between calcification in carotid plaques and clinical ischemic symptoms. J Vasc Surg 2010;51:1015-1025.

24 Eesa M, Hill MD, Al-Khathaami A, Al-Zawahmah M, Sharma P, Menon BK, Tymchuk S, Demchuk AM, Goyal M: Role of CT angiographic plaque morphologic characteristics in addition to stenosis in predicting the symptomatic side in carotid artery disease. AJNR Am J Neuroradiol 2010;31:1254-1260.

25 Kurosaki Y, Yoshida K, Endo H, Chin M, Yamagata S: Association between carotid atherosclerosis plaque with high signal intensity on T1-weighted imaging and subsequent ipsilateral ischemic events. Neurosurgery 2011; 68:62-67; discussion 67.

26 Qiao Y, Etesami M, Astor BC, Zeiler SR, Trout HH 3rd, Wasserman BA: Carotid plaque neovascularization and hemorrhage detected by MR imaging are associated with recent cerebrovascular ischemic events. AJNR Am J Neuroradiol 2012;33:755-760. 
27 Wintermark M, Arora S, Tong E, Vittinghoff E, Lau BC, Chien JD, Dillon WP, Saloner D: Carotid plaque computed tomography imaging in stroke and nonstroke patients. Ann Neurol 2008;64:149-157.

28 Saba L, Sanfilippo R, Pirisi R, Pascalis L, Montisci R, Mallarini G: Multidetector-row CT angiography in the study of atherosclerotic carotid arteries. Neuroradiology 2007;49: 623-637.

29 Saba L, Sanfilippo R, Pascalis L, Montisci R, Caddeo G, Mallarini G: Carotid artery wall thickness and ischemic symptoms: evaluation using multi-detector-row CT angiography. Eur Radiol 2008;18:1962-1971.

-30 Romero JM, Babiarz LS, Forero NP, Murphy EK, Schaefer PW, Gonzalez RG, Lev MH: Arterial wall enhancement overlying carotid plaque on CT angiography correlates with symptoms in patients with high grade stenosis. Stroke 2009;40:1894-1896.
1 Raman G, Moorthy D, Hadar N, Dahabreh IJ, O'Donnell TF, Thaler DE, Feldmann E, Lau J, Kitsios GD: Management strategies for asymptomatic carotid stenosis: a systematic review and meta-analysis. Ann Intern Med 2013;158:676-685.

32 Gupta A, Kesavabhotla K, Baradaran H, Kamel H, Pandya A, Giambrone AE, Wright D, Pain KJ, Mtui EE, Suri JS, Sanelli PC, Mushlin AI: Plaque echolucency and stroke risk in asymptomatic carotid stenosis: a systematic review and meta-analysis. Stroke 2015;46:9197.
3 Abbott AL, Nicolaides AN: Improving outcomes in patients with carotid stenosis: call for better research opportunities and standards. Stroke 2015;46:7-8.

34 Caplan LR, Hennerici M: Impaired clearance of emboli (washout) is an important link between hypoperfusion, embolism, and ischemic stroke. Arch Neurol 1998;55:14751482.

35 Kwee RM, Truijman MT, van Oostenbrugge RJ, Mess WH, Prins MH, Franke CL, Korten AG, Wildberger JE, Kooi ME: Longitudinal MRI study on the natural history of carotid artery plaques in symptomatic patients. PLoS One 2012;7:e42472.

- 36 Hosseini AA, Kandiyil N, Macsweeney ST, Altaf N, Auer DP: Carotid plaque hemorrhage on magnetic resonance imaging strongly predicts recurrent ischemia and stroke. Ann Neurol 2013;73:774-784. 